\title{
Parâmetros fisiológicos de tabagistas em tratamento na Paraíba
}

\author{
Physiological parameters of smokers in treatment in Paraíba \\ Parámetros fisiológicos de humos en tratamiento en Paraíba
}

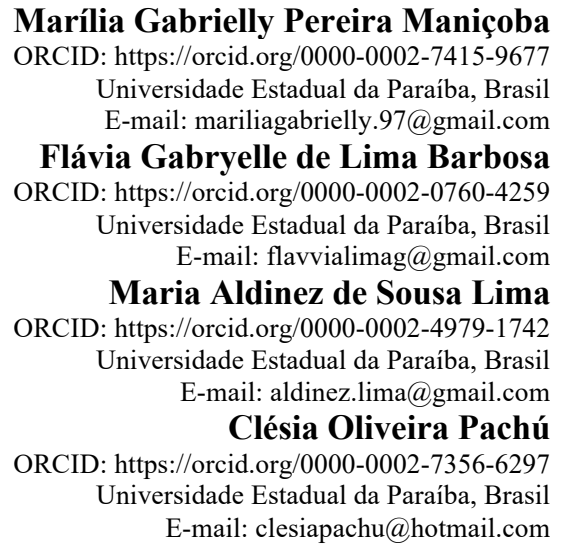

\section{Resumo}

O tabagismo é uma pandemia que além da nicotina envolve outras substâncias, resultando em morbimortalidades. Deste modo, objetivou-se avaliar os parâmetros fisiológicos de tabagistas em tratamento na Paraíba. Trata-se de estudo descritivo com 100 tabagistas, de ambos os sexos e idade superior a 18 anos, voluntários do tratamento no Programa Multidisciplinar de Tratamento de Tabagistas da Universidade Federal de Campina Grande, Paraíba, de abril a setembro de 2018. Na primeira etapa, os tabagistas assistiram a palestras acerca do papel de todas as ou das equipes no tratamento (Medicina, Farmácia, Psicologia, Nutrição, Educação física e Odontologia). Posteriormente, a equipe de farmácia efetuou a Consulta Farmacêutica (perfil socioeconômico e parâmetros fisiológicos). Dos assistidos, 65 e $35 \%$ eram do sexo feminino e masculino, respectivamente. A faixa etária prevalente foi de pessoas entre 46-60 anos (54\%). Quanto aos parâmetros fisiológicos: $47 \%$ eram pré-hipertensos; glicemia plasmática casual de $87 \%$ foi menor que $200 \mathrm{mg} / \mathrm{dL}$; $9 \%$ do público apresentou a relação circunferência-quadril maior que $0,90 \mathrm{~cm}$ e $20 \%$ do público feminino acima de 0,85 cm; o nível de gordura visceral de 59\% dos assistidos estava normal. Os resultados da avaliação dos parâmetros fisiológicos dos tabagistas revelaram sérios riscos, principalmente em relação aos vasos sanguíneos, entendendo que a prática tabagista somada a elevação da pressão arterial apresenta pior prognóstico cardiovascular, conduz a alterações e enrijecimento das estruturas arteriais, podendo dificultar o bombeamento sanguíneo, com grande risco de doenças cardíacas e desenvolvimento de diabetes e câncer. Ademais, este estudo contribuiu com a promoção da saúde dos tabagistas, minimizando os riscos de problemas relacionados ao tabaco utilizando parâmetros fisiológicos como norte da atenção farmacêutica.

Palavras-chave: Atenção farmacêutica; Tabagismo; Bioimpedância; Promoção da saúde.

\begin{abstract}
Smoking is a pandemic in addition that beyond nicotine involves other substances, resulting in morbidity and mortality. This way, the objective of this study was to evaluate the physiological parameters of smokers undergoing treatment in Paraíba. This is a descriptive study with 100 smokers, of both sexes and over the age of 18, volunteers for treatment in the Multidisciplinary Program for the Treatment of Smokers at the Federal University of Campina Grande, Paraíba, from April to September 2018. In the first stage, smokers attended lectures about the role of all teams in the treatment (Medicine, Pharmacy, Psychology, Nutrition, Physical Education, and Dentistry). Subsequently, the pharmacy team conducted the Pharmaceutical Consultation (socioeconomic profile and physiological parameters). Of those assisted, 65 and 35\% were female and male, respectively. The prevalent age group was between 46-60 years old (54\%). As for physiological parameters: $47 \%$ were pre-hypertensive; casual plasma glucose of $87 \%$ was less than $200 \mathrm{mg} / \mathrm{dL}$; $9 \%$ of the public had a circumference-hip ratio greater than $0.90 \mathrm{~cm}$ and $20 \%$ of the female public above $0.85 \mathrm{~cm}$; the level of visceral fat in $59 \%$ of those assisted was normal. The results of the smokers' physiological parameters showed serious risks, especially in relation to blood vessels, understanding that the practice of smoking leads to alterations and stiffening of the structures in the arteries, which can make it difficult to pump blood, with a great risk of heart disease and development of diabetes and cancer. In addition, this study contributed to the promotion of smoker's health by
\end{abstract}


minimizing the risks of problems related to tobacco using physiological parameters such as the north of pharmaceutical care.

Keywords: Pharmaceutical attention; Smoking; Bioimpedance; Health promotion.

\begin{abstract}
Resumen
El tabaquismo es una pandemia que además de la nicotina involucra otras sustancias, lo que resulta en morbilidad y mortalidad. Así, el objetivo fue evaluar los parámetros fisiológicos de fumadores en tratamiento en Paraíba. Se trata de un estudio descriptivo con 100 fumadores, de ambos sexos y mayores de 18 años, voluntarios de tratamiento en el Programa Multidisciplinario de Tratamiento de Fumadores de la Universidad Federal de Campina Grande, Paraíba, de abril a septiembre de 2018. En una primera etapa, los fumadores asistieron a charlas sobre el papel de todos los equipos en el tratamiento (Medicina, Farmacia, Psicología, Nutrición, Educación Física y Odontología). Posteriormente, el equipo de farmacia realizó la Consulta Farmacéutica (perfil socioeconómico y parámetros físiológicos). De los asistidos, el 65 y el $35 \%$ eran mujeres y hombres, respectivamente. El grupo de edad prevalente fue el de personas entre 46 y 60 años (54\%). En cuanto a los parámetros fisiológicos: el $47 \%$ eran prehipertensos; la glucosa plasmática casual del $87 \%$ fue inferior a $200 \mathrm{mg} / \mathrm{dL}$; El 9\% del público tenía una relación circunferencia-cadera superior a $0,90 \mathrm{~cm}$ y el $20 \%$ del público femenino tenía más de $0,85 \mathrm{~cm}$; el nivel de grasa visceral del $59 \%$ de los asistidos fue normal. Los resultados de los parámetros fisiológicos de los fumadores revelaron graves riesgos, especialmente en relación con los vasos sanguíneos, entendiendo que el tabaquismo provoca cambios y endurecimiento de las estructuras arteriales, lo que puede dificultar el bombeo sanguíneo, con alto riesgo de cardiopatía y desarrollo de diabetes y cáncer. Además, este estudio contribuyó a promover la salud de los fumadores, minimizando los riesgos de problemas relacionados con el tabaco utilizando parámetros fisiológicos como pauta de atención farmacéutica.
\end{abstract}

Palabras clave: Atención farmacéutica; De fumar; Bioimpedancia; Promoción de la salud.

\title{
1. Introdução
}

A dependência da nicotina é considerada a principal causa de morte evitável em todo o mundo. Assim, afirma-se que o tabagismo representa o ato de consumir tabaco, ou a intoxicação causada pelo seu uso, cuja droga ou princípio ativo é a nicotina" (Silvano, 2012). Estima-se que um terço da população mundial, cerca de 1,2 bilhões de pessoas, seja composta por fumantes (Organização Mundial da Saúde [OMS], 2014).

Nesse contexto, o tabagismo tem sido considerado uma pandemia mundial devido ao número de usuários dependentes da nicotina. Está relacionado a 30\% dos casos de mortalidade de diversos tipos de cânceres: pulmão, laringe, faringe, boca, esôfago, pâncreas, rins, bexiga e colo do útero. A nicotina presente no cigarro causa dependência química similar a drogas como álcool, heroína e cocaína (Instituto Nacional de Câncer [INCA], 2020).

Ao que concerne o processo de envelhecimento, que acarreta diversas alterações fisiológicas, o tabagismo é um fator determinante no decurso deste processo, estando intimamente ligado com a exacerbação de doenças crônicas e aceleração de efeitos deletérios que poderiam ser prevenidos, quando não envolvem heranças genéticas. Os efeitos nocivos do cigarro tornam seus usuários, em sua grande maioria, caracterizados como indivíduos mais susceptíveis em períodos de crise sanitária, como a pandemia da COVID-19, por exemplo. Estudos desenvolvidos nesta área, buscam evidenciar a correlação entre a resposta imunológica frente à COVID-19 de indivíduos sadios e fumantes. Mesmo estes mecanismos não sendo totalmente elucidados, as manifestações mais severas da doença se fazem presentes em maior número nos indivíduos que fazem uso do cigarro quando comparados à indivíduos sadios (Aires et a 2019; Pessoa et al., 2020).

A dependência desencadeada pela nicotina está relacionada com a ligação desta substância aos receptores nicotínicos presentes na região do sistema de recompensa cerebral (SRC), resultando na liberação da dopamina, uma catecolamina responsável por proporcionar ao indivíduo sensações de prazer, melhora na atenção e aumento da capacidade de memória, por exemplo (Sarmento et al., 2019).

Além da nicotina, o cigarro apresenta substâncias como monóxido de carbono, alcatrão e naftalina. Quando absorvidas podem provocar doenças cardiovasculares (angina, infarto do miocárdio), câncer e doenças pulmonares obstrutivas crônicas (DPOC) como enfisema e bronquite. $\mathrm{O}$ tabagismo ainda pode causar impotência sexual no homem, complicações na gravidez, aneurismas arteriais, úlceras do aparelho digestivo e infecções respiratórias (Instituto Nacional de Câncer [INCA], 2020). 
Conhecendo o metabolismo das substâncias presentes no cigarro, estas quando absorvidas podem acarretar em alterações nos parâmetros fisiológicos como: aumento da Pressão Arterial (PA), aumento da viscosidade do sangue, diminuição da concentração de oxigênio no sangue, diminuição do Índice de Massa Corporal (IMC), aumento da Circunferência Abdominal (CA) e diminuição da Circunferência do Quadril (CQ).

Para contrapor aos prejuízos à saúde, diversas intervenções farmacológicas estão sendo utilizadas no tratamento do tabagismo. Entre elas, destaca-se o uso de Bupropiona, um antidepressivo atípico de ação lenta. Este, por sua vez, inibe a recaptação da dopamina e noradrenalina, e serve de antagonista de receptores nicotínicos, diminuindo os sintomas relacionados à síndrome da abstinência e à vontade de fumar (Balbani \& Montovani, 2005).

Diante do grande potencial maléfico do tabagismo nos fumantes, tanto ativos quanto passivos, e, nas alterações dos parâmetros fisiológicos provocados, vislumbra-se à redução dos malefícios que o tabaco traz aos pacientes e contribuições para melhoria da qualidade de vida desses pacientes, inclusive entre os indivíduos que já desenvolveram alguma doença. O presente estudo objetivou avaliar os parâmetros fisiológicos de tabagistas que iniciaram tratamento em um hospital público da Paraíba.

\section{Fundamentação teórica}

\subsection{Doenças tabaco-relacionadas}

O tabagismo é considerado pela Organização Mundial de Saúde como um dos maiores problemas de saúde pública da atualidade e a principal causa evitável de morte. Responsável por mais de 8 milhões de óbitos anuais no mundo, sendo 7 milhões dessas mortes resultam do uso direto deste produto, enquanto cerca de 1,2 milhão é o resultado de não-fumantes expostos ao fumo passivo (Instituto Nacional de Câncer [INCA], 2021).

Encontra-se na literatura associação do tabagismo com inúmeras doenças, tais como as neoplasias de pulmão, mesmo que para usuários passivos (90-95\%), boca, laringe, faringe, esôfago ( $80 \%$ ), bexiga e rins (25\%), pâncreas, estômago, colo do útero e cólon (25 a 30\%). Entre as doenças cardiovasculares, pode-se citar a doença coronariana (risco 4 vezes maior), o acidente vascular cerebral (risco duas vezes maior), o aneurisma de aorta abdominal (Bittencourt et al., 2017).

Já, com relação às doenças respiratórias, destaca-se a Doença Pulmonar Obstrutiva Crônica (DPOC), relacionada ao tabagismo em $80 \%$ a $90 \%$ dos casos (Benowitz, 2001; Alves, 2006). Outras doenças em diversos órgãos e sistemas, como as gastrites, úlceras e doença do refluxo; complicações do diabetes, hipotireoidismo e osteoporose; dificuldade de cicatrização e complicações pós-cirúrgicas; impotência sexual e complicações materno-fetais tem sido cada vez mais associadas ao tabagismo (Alves, 2006).

\subsection{Impacto do tabagismo na economia}

Observando a participação da indústria do tabaco na arrecadação de impostos para a União, o tabagismo contribui para a economia tanto na geração de riqueza como em gastos em saúde. No estudo concluído por Pinto et al. (2012), realizou-se um levantamento dos gastos diretos do Ministério da Saúde com complicações tabaco-relacionado, que consiste na prestação de serviço para diagnóstico e tratamento de até 2 anos financiados pelo Sistema Único de Saúde (SUS). O resultado de tal pesquisa indicou que 30\% do orçamento do Fundo Nacional da Saúde de 2011 foi utilizado em decorrência do tabagismo, correspondendo a quase 20,6 bilhões de reais. Mesmo considerando a contribuição da indústria do tabaco para a Receita Federal em detrimento para tal problema de saúde pública, a arrecadação de impostos do mesmo ano sobre o produto se restringe a 6,3 bilhões, representando assim, um valor cerca de 3,5 vezes menor que o impacto direto causado pelo cigarro (Pinto et al., 2012).

Tendo em vista a dimensão do grave problema de saúde e econômico, o surgimento do Plano Nacional de Controle do Tabagismo (PNCT), criado pelo Ministério da Saúde na década de 90, responsabilizou na difusão de informações acerca dos problemas acarretados à saúde, iniciando dessa forma, a participação governamental neste controle (INCA, 2011). Outras 
organizações não governamentais e entidades de saúde participam intensamente tanto para garantir o ambiente livre de fumo quanto na promoção da abstinência em usuários. Afinal, parar de fumar aumenta a expectativa e a qualidade de vida em qualquer faixa etária, inclusive entre os indivíduos que já desenvolveram alguma doença (INCA, 2011).

O governo brasileiro lançou várias medidas para impor de forma contrária às estratégias utilizadas pela indústria do cigarro, como proibição à exibição de comerciais incentivando o seu uso, obrigação da presença de avisos informativos e valor mínimo de venda em carteiras de cigarro, dentre outros. Em dezembro de 2011, a presidente Dilma Rousseff sancionou a lei que proíbe o fumo em locais fechados e coletivos. O projeto, originário da medida provisória 540/2011, que altera a legislação sobre o fumo, foi aprovado pelos senadores no final de novembro de 2011. Porém, apenas em 2014, com o Decreto 8.262, ficou proibido o uso de cigarros, cigarrilhas, charutos e cachimbos em ambientes fechados, os chamados "fumódromos", tanto privados quanto públicos.

\subsection{Alteração de parâmetros fisiológicos}

Segundo Kumar et al. (2010), a quantidade de substâncias altamente nocivas existentes no cigarro está entre 2.000 e 4.000, sendo algumas consideradas cancerígenas. Estas, como a nicotina, alcatrão, fenol e naftalina, quando absorvidas, provocam efeitos nos parâmetros fisiológicos, comprometendo a saúde humana. Estudos têm comprovado que, ao serem absorvidas, tais substâncias ocasionam no ser humano: tremores, falhas respiratórias, efeitos antidiuréticos, tosse, dispneia e infecções nas vias aéreas superiores. A saúde do corpo também sofre com a elevação da frequência cardíaca e da pressão arterial, diminuição do oxigênio no sangue e alterações celulares, possivelmente, resultarão em câncer (Instituto Nacional de Câncer [INCA], 2021).

O Instituto Nacional de Câncer (INCA) relata que, ao parar de fumar, o usuário pode relatar dor de cabeça, agressividade, tosse, alteração do sono e outros sintomas. Entretanto, o corpo estimula respostas positivas, como por exemplo, após 2 horas não haverá mais nicotina circulando no sangue; após 8 horas, o nível de oxigênio no sangue se normaliza; após 1 ano, o risco de morte por infarto do miocárdio é reduzido à metade; e após 10 anos, o risco de sofrer infarto será igual ao das pessoas que nunca fumaram (Instituto Nacional de Câncer [INCA], 2021).

O tabaco e os comportamentos tabagistas são elementos comuns nas sociedades, figurando como elemento de status no passado, tendo transformado o hábito em uma prática concretizada como cultura. Felizmente, essa ideia está mudando, e fumar não é mais visto como algo agradável e passível de ser invejado, mas sim, como um ato destoante dos atuais anseios da sociedade mais bem informada acerca do que é saudável.

Contudo, é bastante conhecida a dificuldade de abandonar o hábito tabagista, aumentando a dificuldade quanto maior o tempo de consumo e a intensidade do uso da droga. A dependência causada pelo hábito tabagista está associada a fatores tanto químicos - proporcionados por substância psicoativa - como por fatores psicológicos - quando as atividades da vida cotidiana estão associadas ao uso da droga. Assim, a participação é fundamental tanto no aconselhamento, quanto na indicação de tratamento eficaz, medicamentoso ou não, para induzir a abstinência deste mal (Ferreira et al., 2015).

Para ajudar os pacientes em tratamento do tabagismo, na maioria dos casos são recomendadas intervenções farmacológicas. O mais recomendado é o uso da Bupropiona, um antidepressivo bem tolerado, com baixos relatos de ocorrência de efeitos colaterais (Moreno et al., 1999).

A Bupropiona, inicialmente, não era utilizada no tratamento do tabagismo. Tratava-se de um antidepressivo comum, e durante a realização de seus ensaios clínicos para a verificação de sua eficácia antidepressiva, alguns pacientes relataram uma menor intensidade no desejo de fumar. Após a aprovação da Bupropiona como tratamento depressivo, iniciaram-se os estudos da droga para comprovar o seu tratamento do tabagismo. E no ano de 1998, foi liberado o seu uso para tratamento da dependência à nicotina (Laranjeira \& Alves, 2002). 
Ressalta-se que o mecanismo fisiológico de ação da Bupropiona parece estar ligado a sua ação inibidora da recaptação da dopamina e noradrenalina. A nicotina eleva os níveis de dopamina cerebral em áreas associadas ao efeito reforçador de drogas. Acredita-se que a ação dopaminérgica do fármaco reduziria a propriedade reforçadora da droga e sua atividade noradrenérgica reduzindo os sintomas de abstinência (Laranjeira \& Alves, 2002).

$\mathrm{O}$ tratamento com Bupropiona deve iniciar uma semana antes da abstinência e não deve ultrapassar a dose total diária de $300 \mathrm{mg}$. Tendo início com doses diárias de $150 \mathrm{mg}$ uma vez ao dia por quatro dias consecutivos. Se o tratamento for bem tolerado pelo organismo, as doses diárias devem ser de $300 \mathrm{mg}$, sendo fracionadas em duas doses de $150 \mathrm{mg}$, com um intervalo de pelo menos oito horas entre as duas doses. Esse tratamento deve ser contínuo entre três e quatro meses para obtenção de resultados terapêuticos positivos (Laranjeira \& Alves, 2002).

Apesar de ser bem tolerado, o tratamento com Bupropiona pode apresentar efeitos colaterais. Entre eles, convulsões ou ataques epilépticos, reações alérgicas, distúrbios do sono, cefaleia, febre, tontura, coceira, sudorese, erupção cutânea e urticária. Além de tremores, calafrios; ansiedade; boca seca, dor abdominal, enjoo, vômito, constipação, perda de apetite; mudanças na pressão sanguínea, rubor e transtornos visuais (“Bupropiona - Bula de Bupropiona”, 2020).

\section{Metodologia}

O Projeto Multidisciplinar de Tratamento do Tabagismo representa uma parceria formada pela Universidade Federal de Campina Grande (UFCG), Universidade Estadual da Paraíba (UEPB) e Universidade Maurício de Nassau (UNINASSAU) sendo constituído por equipe multidisciplinar (Medicina, Farmácia, Educação Física, Psicologia, Odontologia e Nutrição).

Foi desenvolvido a partir de uma pesquisa de caráter descritiva. Segundo Gil (2010), pesquisa descritiva, visa a descrição das características de determinada população ou fenômeno ou estabelecimento de relações entre variáveis.

O local de realização do presente estudo foi o Hospital Alcides Carneiro (HUAC), da Universidade Federal de Campina Grande. O presente estudo foi desenvolvido no período de abril a setembro de 2018.

Foram pesquisados 100 tabagistas voluntários, de ambos os sexos e idade superior a 18 anos, disponíveis às sextasfeiras à tarde para realização do tratamento. Estes foram recebidos nas primeiras sextas-feiras do mês, o retorno se dava de 15 em 15 dias enquanto o tratamento durava 3 meses. Os assistidos eram divididos em quatro grupos para facilitar o acolhimento das equipes durante o retorno.

Acerca das considerações éticas, o projeto foi aprovado pelo Comitê de Ética em Pesquisa da Universidade Federal de Campina Grande $\mathrm{n}^{\mathrm{o}}$ 77465017.0.0000.5182. Quanto aos participantes do estudo, foi explicado o propósito, objetivos e procedimentos da pesquisa e solicitado a assinatura de um Termo de Consentimento Livre e Esclarecido.

$\mathrm{O}$ tratamento do tabagismo se iniciava com uma palestra com todas as equipes, onde era apresentado todo o processo de tratamento e o papel de cada equipe. Posteriormente, encontros multidisciplinares quinzenais eram realizados, e nesse primeiro momento, a equipe de farmácia com o auxílio de um formulário realizava a entrevista individual, acerca do perfil socioeconômico, verificação dos parâmetros fisiológicos atuais, orientação de como utilizar a Bupropiona e dispensação com quantidade que deve durar até o próximo encontro, ou seja, 15 dias.

A cada retorno os indivíduos recebiam 25 comprimidos de Bupropiona, medicamento usado para o auxílio dos sintomas de abstinência apresentados, deveriam tomá-lo uma vez ao dia, nos três primeiros dias, após o café-da-manhã, segundo a prescrição da equipe de medicina (UFCG). A partir do quarto dia o paciente deve passar a tomar dois comprimidos por dia, um após o café-da-manhã e outro antes das $16: 00$ h, visto que há relatos de insônia em paciente que administram o segundo comprimido em um horário mais tardio.

Dava-se atenção aos sintomas dos pacientes e os parâmetros fisiológicos, pois Bupropiona causa um aumento da PA, então não se dispensava para pacientes que apresentavam a pressão arterial acima de 130 x $90 \mathrm{mmHg}$, exceto o hipertenso 
controlado. Também não podia ser dispensado para mulheres grávidas, que desejavam se tornar grávidas ou que estavam amamentando, para pessoas que apresentavam convulsões, algum tipo de arritmia cardíaca, entre outros.

Os valores de referências das seguintes variáveis: Índice de massa corporal, porcentagem de gordura corporal e nível de gordura visceral, foram de acordo com tabelas do manual de instrução da balança digital de Bioimpedância. Os dados utilizados para avaliar a pressão arterial foi baseado na $7^{\mathrm{a}}$ Diretriz Brasileira de Hipertensão Arterial. A glicemia plasmática casual foi medida por glicosímetro simples e, a SPO2 por oxímetro de dedo.

Salienta-se que os dados apresentados neste artigo são dos parâmetros fisiológicos de tabagistas que iriam iniciar o tratamento, ou seja, sem intervenção farmacológica.

Os dados coletados foram tabelados e analisados por meio de estatística descritiva.

\section{Resultados e Discussão}

No período de abril a setembro de 2018 o Programa Multidisciplinar de Tratamento do Tabagismo (PMTT) atendeu 100 tabagistas voluntários. Na tabela 1, observa-se que 65 e 35\% pertenciam ao sexo feminino e masculino, respectivamente. Em relação à faixa etária, a idade predominante de ambos os sexos foi de 46 a 60 anos, correspondendo (54\%), seguidos pela faixa etária de 36 a 45 anos (17\%), 26 a 35 anos (15\%), acima de 60 anos (8\%), 18 a 25 anos (5\%), e idade não informada (1\%).

Podemos observar que a grande maioria dos tabagistas pertenciam ao sexo feminino e, predominância de 46 a 60 anos de idade dos pesquisados. Estes dados assemelham-se a um estudo realizado por Santos et al. (2019) em um Centro de Atenção Psicossocial Álcool e outras Drogas-CAPS-AD, de uma cidade do Sul do país. Neste, a idade média para início do tratamento foi de 45 anos para os fumantes que buscaram tratamento para cessação do tabagismo no CAPS, no período de 2013 até o ano de 2016, de forma semelhante ao verificado em outros estudos.

A maior procura das mulheres pelo serviço de saúde pode estar relacionada com o maior cuidado e preocupação que as mesmas têm com a saúde, em comparação aos homens. Segundo Pereira et al. (2015), estudosapontam o risco elevado de vários tipos de cânceres em fumantes do sexo feminino, particularmente câncer de bexiga e de útero, além de aumentar o risco de infertilidade nas mulheres. Além disso, com as mudanças sociais, econômicas e culturais, levando a entrada acentuada das mulheres no mercado de trabalho, a indústria tabagista passou a investir em campanhas publicitárias, com propagandas voltadas à liberdade, independência, auto afirmação, ascensão social e beleza. Iniciando assim, o consumo do tabaco entre mulheres. 
Tabela 1: Distribuição dos tabagistas pesquisados segundo características socioeconômicas.

\begin{tabular}{|c|c|c|}
\hline VARIÁVEIS & $\mathbf{N}$ & $\%$ \\
\hline \multicolumn{3}{|l|}{ Sexo } \\
\hline Feminino & 65 & 65 \\
\hline Masculino & 35 & 35 \\
\hline \multicolumn{3}{|l|}{ Faixa Etária } \\
\hline $18-25$ anos & 5 & 5 \\
\hline $26-35$ anos & 15 & 15 \\
\hline $36-45$ anos & 17 & 17 \\
\hline $46-60$ anos & 54 & 54 \\
\hline Acima de 60 anos & 8 & 8 \\
\hline Não Informado & 1 & 1 \\
\hline \multicolumn{3}{|l|}{ Escolaridade } \\
\hline Analfabeto & 5 & 5 \\
\hline Semianalfabeto & 2 & 2 \\
\hline Primeiro Grau Incompleto & 20 & 20 \\
\hline Primeiro Grau Completo & 14 & 14 \\
\hline Segundo Grau Incompleto & 16 & 16 \\
\hline Segundo Grau Completo & 25 & 25 \\
\hline Nível Superior Incompleto & 5 & 5 \\
\hline Nível Superior Completo & 10 & 10 \\
\hline Outros & 1 & 1 \\
\hline Não Informado & 2 & 2 \\
\hline \multicolumn{3}{|l|}{ Renda } \\
\hline Até 2,5 Salários Mínimos & 74 & 74 \\
\hline De 2-4 Salários Mínimos & 21 & 21 \\
\hline De 10-20 Salários Mínimos & 2 & 2 \\
\hline Não Informado & 3 & 3 \\
\hline
\end{tabular}

Fonte: Autores (2021).

No Brasil, indivíduos com baixo nível de escolaridade têm probabilidade 35 vezes maior de serem fumantes em relação aos indivíduos que possuem o segundo grau completo ou nível superior (De Souza Leal et al., 2017). No presente estudo, a maioria dos avaliados havia cursado o segundo grau completo $25 \%$, seguidos por $20 \%$ com primeiro grau incompleto. De acordo com o Inquérito nacional recente (Vigitel), realizado no ano de 2018 por meio de entrevistas telefônicas a 1.009.074 de pessoas, os menos escolarizados são os que mais fumam, correspondendo a 13\% dos entrevistados com até oito anos de escolaridade.

A renda é uma variável sociodemográfica que relaciona todas as outras já discutidas. O tabagismo aparece consistentemente maior entre os grupos com baixo nível socioeconômico no Brasil e em outros países (Caram et al., 2009). Dos pesquisados, 74\% possuía renda de até 2,5 salários mínimos. Assemelhando-se ao estudo de Bazotti et al. (2015), onde indivíduos pertencentes às faixas de renda mais baixas e com idade entre 30 e 45 anos são os maiores consumidores de tabaco.

De outro modo, em homens e mulheres, o tabagismo aumenta a incidência de infarto do miocárdio e doença arterial coronariana. O fumo causa um aumento agudo da pressão arterial e da frequência cardíaca, provavelmente mediada pela nicotina que age como um agonista adrenérgico, promovendo a liberação local e sistêmica de catecolaminas (dopamina, norepinefrina, vasopressina) (Sousa, 2015).

Classificando a pressão arterial, baseado na $7^{\mathrm{a}}$ Diretriz Brasileira de Hipertensão Arterial, observou-se que 33\% dos sujeitos apresentaram pressão arterial normal, 47\% pré-hipertenso, 10\% hipertensão estágio I, 4\% hipertensão estágio II e, 6\% não foi informado (Tabela 2). Este achado se mostra preocupante visto que a hipertensão arterial se apresenta como um fator de risco fortemente associado a eventos cardiovasculares graves na população mundial e brasileira. 
Tabela 2: Parâmetros fisiológicos atuais dos Pesquisados.

\begin{tabular}{|c|c|c|}
\hline $\begin{array}{l}\text { VARIÁVEIS } \\
\end{array}$ & $\mathbf{N}$ & $\%$ \\
\hline \multicolumn{3}{|l|}{ Pressão Arterial (mmHg) } \\
\hline \multicolumn{3}{|l|}{ Normal: } \\
\hline $\mathrm{PAS} \leq 120 \mathrm{PAD} \leq 80$ & 33 & 33 \\
\hline \multicolumn{3}{|l|}{ Pré-hipertenso: } \\
\hline PAS 121-139 PAD 81-89 & 47 & 47 \\
\hline \multicolumn{3}{|l|}{ Hipertensão Estágio I: } \\
\hline PAS 140-159 PAD 90-99 & 10 & 10 \\
\hline \multicolumn{3}{|l|}{ Hipertensão Estágio II: } \\
\hline $\mathrm{PAS} \geq 160 \mathrm{PAD} \geq 100$ & 4 & 4 \\
\hline Não Informado & 6 & 6 \\
\hline \multicolumn{3}{|c|}{ Glicemia Plasmática Casual } \\
\hline$<200$ & 87 & 87 \\
\hline$\geq 200$ & 7 & 7 \\
\hline Não Informado & 6 & 6 \\
\hline \multicolumn{3}{|l|}{ spO2 } \\
\hline$<95 \%$ & 1 & 1 \\
\hline $95-100 \%$ & 97 & 97 \\
\hline Não Informado & 2 & 2 \\
\hline
\end{tabular}

Fonte: Autores (2021).

Nesse sentido, fumar é prejudicial às células endoteliais vasculares e a combinação de tabagismo e intolerância à glicose tem efeitos adversos graves, podendo conduzir ao desenvolvimento precoce da aterosclerose e aumento da resistência à insulina. Avaliando a glicemia plasmática casual, considerando maior ou igual a $200 \mathrm{mg} / \mathrm{dL}$ variável de risco para desenvolvimento da diabetes tipo 2, dos indivíduos da pesquisa, (87\%) apresentaram glicemia casual menor que $200 \mathrm{mg} / \mathrm{dL}$, (7\%) maior ou igual a $200 \mathrm{mg} / \mathrm{dL}$ e (6\%) não foi informado. Todavia, o percentual de (7\%) de glicemia casual $200 \mathrm{mg} / \mathrm{dL}$ é relevante, principalmente se ocorreu em indivíduos com diabetes já confirmada. Salienta-se que nenhum dos participantes estava com tempo de jejum maior ou igual a 8 horas.

A redução da saturação de oxigênio no sangue tem sido associada ao agravamento de doenças pulmonares preexistentes. Em um estudo realizado por Vold et al. (2014), a redução da SpO2 estava associada a história tabagista, função pulmonar, sexo e idade, Índice de massa corporal (IMC) sendo este à medida que aumentava, a frequência de declínio da SpO2 aumentou. O oposto foi o caso quando o IMC caiu. Essa tendência não foi estatisticamente significativa. Nesse estudo, a maioria dos participantes (97\%) apresentou saturação de oxigênio entre 95-100\%, (1\%) menor que 95\% e (2\%) não foi informado.

Outro aspecto que deve ser observado nos tabagistas é a circunferência abdominal. Segundo o Instituto Nacional de Câncer (INCA, 2019), a obesidade abdominal (circunferência da cintura elevada) se apresenta como indicativo de acumulação de gordura nessa região e representa um importante fator de risco para o desenvolvimento de doenças cardiovasculares, câncer e diabetes. Além disso, sugerem que a associação entre o fumo e a circunferência da cintura elevada seria pelo fato que a nicotina aumenta a resistência insulínica, que por sua vez, está relacionada ao depósito de gordura na região abdominal. Dos indivíduos pesquisados, (7\%) dos homens apresentaram circunferência abdominal maior ou igual a $94 \mathrm{~cm}$ e (7\%) menor que $94 \mathrm{~cm}$. Das mulheres, (20\%) apresentou circunferência abdominal maior ou igual a $80 \mathrm{~cm}$ e $(10 \%)$ menor que $80 \mathrm{~cm}$. Não foi informado a circunferência dos $(56 \%)$ restantes pesquisados.

A relação circunferência quadril (RCQ), calculada dividindo-se a medida da circunferência da cintura em centímetros pela medida da circunferência do quadril em centímetros, (9\%) dos homens apresentaram índice de corte igual ou maior que $0,90 \mathrm{~cm}$ e (5\%) menor que 0,90 cm. Das mulheres, (20\%) apresentaram valor de corte igual ou maior que 0,85 cm e (10\%) menor que $0,85 \mathrm{~cm}$. (56\%) não foi informado. 
Os resultados da relação circunferência quadril são consistentes com o estudo de Faria et al. (2012), em que encontra uma associação positiva entre o tabagismo e a gordura abdominal. Essa associação foi encontrada tanto para circunferência da cintura como para RCQ em fumantes de mais de 5 cigarros por dia, independentemente de outros fatores. Apresentam ainda um estudo realizado em Norfolk, Reino Unido, onde aqueles com maior exposição cumulativa ao tabaco, maior número de anos-maço apresentou maior RCQ que os não fumantes, mesmo após o ajuste para outros fatores. Além disso, a gordura abdominal é um indicador de gordura visceral, que tem perfil aterogênico, causando complicações metabólicas e aumentando o risco de morte (Faria et al., 2012).

$\mathrm{Na}$ avaliação do IMC, indicador da obesidade generalizada, (39\%) dos pacientes apresentou peso adequado, (37\%) sobrepeso, (19\%) obesidade e (5\%) estavam abaixo do peso (Tabela 3). Frequentemente, fumantes apresentam menores índices de massa corporal, quando comparados a não fumantes devido a ação da dopamina que causa aumento de neurotransmissores, como dopamina e serotonina, que são substâncias inibidoras de apetite. No processo de tratamento para cessação do cigarro, o aumento de peso corporal é notório tanto em homens como em mulheres, sendo este uma das dificuldades apontadas para a manutenção da abstinência, principalmente em mulheres. O IMC tem sido também utilizado na avaliação de risco cardiovascular e nas decisões terapêuticas relacionadas às situações clínicas associadas. 
Tabela 3: Dados dos pesquisados obtido por bioimpedância.

\begin{tabular}{lcc}
\hline \multicolumn{1}{c}{ VARIÁVEIS } & $\mathbf{N}$ & $\mathbf{\%}$ \\
\hline $\begin{array}{l}\text { Índice de Massa Corporal } \\
\text { Baixo Peso } \\
<18,5\end{array}$ & 5 & 5 \\
$\begin{array}{l}\text { Peso Adequado } \\
\geq 18,5<25\end{array}$ & 39 & 39 \\
Sobrepeso & & 37 \\
$\geq 25<30$ & 37 & 19 \\
Obesidade & & \\
$\geq 30$ & 19 &
\end{tabular}

\section{Porcentagem de Gordura Corporal}

\section{(-) Baixo (0) Normal (+) Alto (++) Muito}

Alto

Feminino:

20-39 anos

$(-)<21,0$

(0) $21,0-32,9$

(+) $33,0-38,9$

$(++) \geq 39,0$

$40-59$ anos

$(-)<23,0$

(0) $23,0-33,9$

(+) $34,0-39,9$

$(++) \geq 40,0$

60-79 anos

$(-)<24,0$

(0) 24,0 - 35,9

(+) $36,0-41,9$

$(++) \geq 42,0$

$\begin{array}{cc}0 & 0 \\ 2 & 2 \\ 1 & 1 \\ 5 & 5 \\ & \\ 2 & \\ 6 & 2 \\ 10 & 6 \\ 24 & 10 \\ & 24 \\ 1 & \\ 2 & 1 \\ 3 & 2 \\ 2 & 3 \\ & \\ & \\ & \\ & \end{array}$

\section{Masculino:}

20-39 anos

$(-)<8,0$
(0) $8,0-19,9$
(+) $20,0-24,9$

(+) $20,0-24,9$

$40-59$ anos

$(-)<11,0$

(0) $11,0-21,9$

(+) $22,0-27,9$

$(++) \geq 28,0$

60-79 anos

$(-)<13,0$

(0) $13,0-24,9$

(+) $25,0-29,9$

$(++) \geq 30,0$

Não informado

$\begin{array}{ll}0 & 0 \\ 5 & 5 \\ 2 & 2 \\ 6 & 6\end{array}$

Nível de Gordura Visceral

\begin{tabular}{lcc}
$(0) 1-9$ & 59 & 59 \\
$(+) 10-14$ & 19 & 19 \\
$(++) 15-30$ & 4 & 4 \\
Não Informado & 18 & 18 \\
\hline
\end{tabular}

Fonte: Autores (2021).

O tabagismo aparece como precursor de diversas doenças, principalmente cardiovasculares, somado ao acúmulo intraabdominal de gordura corporal, pode conferir um maior risco. Avaliando a porcentagem de gordura corporal dos assistidos, observa-se que as mulheres prevalecem numa porcentagem alta para sua idade, enquanto os homens variam em normal e muito alto para idade. 
Embora os fumantes comumente apresentem um IMC mais baixo em relação aos não fumantes, o fumo pode favorecer o acúmulo de gordura corporal abdominal (Clair et al., 2011). Em estudo transversal de base populacional realizado na Suíça, a circunferência da cintura média, porcentagem de gordura corporal e índice de massa corporal em fumantes atuais era mais baixa em comparação com os não fumantes, porém essas variáveis aumentaram com os cigarros fumados por dia entre os fumantes, principalmente em mulheres.

Há crescente evidência que o tabagismo ao mesmo tempo que faz diminuir o IMC conduz a uma maior acumulação de gordura a nível visceral e a um aumento da insulina-resistência (Violante, 2011). De fato, há evidências seguras de que o tabagismo aumenta o risco de obesidade central e pode causar ou agravar a Síndrome Metabólica em fumadores, caracterizada, na sua plenitude, por hipertensão, HDL-C baixo, triglicerídeos alto e diabetes mellittus.

Estudos realizados em uma população de tabagista comprovaram que estes apresentavam características de síndrome de resistência à insulina, incluindo colesterol HDL baixo, níveis elevados de triglicerídeos, elevações nos níveis de VLDL, glicemias em jejum elevadas, aumento do inibidor do ativador do plasminogênio e microalbuminúria (Kong et al., 2001). Han et al. (2006) afirmam que níveis altos de tecido visceral adiposo estão relacionados com o maior risco de Síndrome Metabólica, Diabetes e Doenças Cardiovasculares. Dos sujeitos pesquisados, 59, 19, 4 e 18\% possuem níveis de gordura visceral: normal, alto, muito alto e não foi informado, respectivamente.

Vale salientar que, o número de variáveis com valor não informado, na maioria dos casos, foi devido a aspectos vinculados ao paciente, seja recusar fazer a medição ou por ter havido falta de paciência.

\section{Conclusão}

Os dados da avaliação dos parâmetros fisiológicos dos pesquisados revelam sérios riscos, principalmente em relação aos vasos sanguíneos, pois a prática tabagista, somada a elevação da pressão arterial, conduz a alterações e enrijecimento das estruturas das artérias, podendo dificultar o bombeamento do sangue. A porcentagem alta de indivíduos pré- hipertensos, circunferência abdominal e de gordura corporal elevada traz uma preocupação e cuidado maior com esses pacientes. Nestes sujeitos o risco de doenças cardíacas, desenvolvimento de diabetes e câncer são altíssimas.

É de extrema importância o acompanhamento nutricional e físico desses pacientes durante e após o tratamento para cessação do cigarro. O IMC adequado, CA e CQ normais, podem garantir melhor qualidade de vida e diminuir os riscos de desenvolver alguma doença tabaco-relacionada, já que a maioria se encontram na condição de tabagistas a anos e possui algum parâmetro fisiológico irregular.

Embora o Brasil possua políticas públicas acerca do controle de tabagismo, a ausência de fiscalização nos pontos comerciais de venda, sinalização e informação em ambientes públicos e privados, não têm levado a efetivação dessas políticas. Cabe aos órgãos governamentais proteger seus cidadãos do perigo do fumo, por meio de leis mais abrangentes contra o tabaco, que incluam todos os locais de trabalho e áreas públicas. Assim, diminuindo, os gastos no sistema de saúde e garantindo saúde para população, visto que, parar de fumar se traduz em benefícios, em qualquer idade.

Por fim, acreditamos ter contribuído na promoção da saúde dos tabagistas minimizando os riscos de problemas relacionados ao tabaco utilizando parâmetros físiológicos como o norte da atenção farmacêutica.

\section{Referências}

Alessi, A., Feitosa, A. D. \& Coelho, E. B (2016) $7^{\text {a }}$ Diretriz Brasileira de Hipertensão Arterial: Capítulo 2 - Diagnóstico e Classificação. Arquivo Brasileiro de Cardiologia, 107, 7

Aires, I. O., Sousa, L. L. C. de, Sousa, D. J. M. de, Araújo, D. S. C. de, Oliveira, I. K. F., \& Alencar, M. do S. S. (2019). Consumo alimentar, estilo de vida e sua influência no processo de envelhecimento. Research, Society and Development, $8(11)$ 
Alves, H.N.P. (2006). Tabagismo: Tratado de Clínica Médica. Roca, p. 2501-2504.

Balbani, A., \& Montovani, J. (2005). Métodos para abandono do tabagismo e tratamento da dependência da nicotina. Revista Brasileira De Otorrinolaringologia 71 (6), 820-827. https://doi.org/10.1590/s0034-72992005000600021

Bazotti, A., Finokiet, M, Conti, I.L., França, M.T.A. \& Waquil, P.D. (2016). Tabagismo e pobreza no Brasil: uma análise do perfil da população tabagista a partir da POF 2008-2009. Ciência e Saúde coletiva, 21 (1), 45-52.

Benowitz, N. L. (2001). Tabaco: Tratado de Medicina Interna. (21a ed.), Guanabara-Koogan S.A, 39-43.

Bittencourt, C. P., Abreu, M. C., Souza, T. de F., Hot, A. D., Partata, A. K. (2017). Tabagismo e sua relação com o desenvolvimento de câncer. Revista Científica do ITPAC, $10(1)$.

Bup: cloridrato de bupropiona: comprimidos 150mg. (2017). (Pereira, M. B., Farmacêutica). Eurofarma.

Caram, L. M. O., Ferrari R., Tanni S. E., Coelho L. S., Godoy I., Martin R. S. S. \& Godoy 1. (2009). Perfil de fumantes atendidos em serviço público para tratamento do tabagismo. Jornal Brasileiro de Pneumologia, 35, 980-985

Clair, C., Chiolero, A., Faeh, D., Cornuz, J., Marques-Vidal, P., Paccaud, F., Mooser, V., Waeber, G., Vollenweider, P. (2011, janeiro 11). Dose-dependent positive association between cigarette smoking, abdominal obesity and body fat: cross-sectional data from a population-based survey. BMC Public Health 11 , n. 23

Chatkin, R. \& Chatkin, J. M. (2007). Tabagismo e variação ponderal: a fisiopatologia e genética podem explicar esta associação? Jornal Brasileiro de Pneumologia. 1-6.

De Souza Leal, A., Silveira, A., Coelho Fiqueira Freire, A., Soares Santos, A., Ramos, D., \& Cipulo Ramos, E. et al. (2017). Características sóciodemográficas e padrão de consumo tabagístico de sexo que procuram atendimento em programas de cessação. O Mundo Da Saúde, 41 (2), 163-169. https://doi.org/10.15343/0104-7809.20174102163169

Faria, C. S., Botelho, C., Silva, R. M. V. G. \& Ferreira, M. G. (2012). Tabagismo e obesidade abdominal em doadores de sangue. Jornal Brasileiro de Pneumologia, 38 (3), 356-363.

Ferraz, L., Busato M. A., Teo, C. R. P. A., Matos T. \& Lieshout, B. (2015). Tabagismo: Motivos da cessação e da recaída na população de um Nasf. Revista Brasileira de Geografia Médica e da Saúde, Hygeia, 127-137.

Ferreira, A., Borba, L., Capistrano, F., Czarnobay, J., \& Maftum, M. (2015). Fatores Que Interferem Na Conformidade Do Paciente Com Tratamento De Dependência Química: Percepções De Profissionais De Saúde. REME: Revista Mineira De Enfermagem, 19 (2). https://doi.org/10.5935/1415-2762.20150032

Gama, G. G. G., Mussi, F. C., Mendes, A.S. \& Guimarães, A. C. (2010). (Des)controle de parâmetros clínicos e antropométricos em indivíduos com doença arterial coronária. Revista da Escola de Enfermagem da USP, 624-631

Gil, A. C. (2010). Como elaborar projetos de pesquisa. (5a ed.), Atlas.

Han, T. S., Sattar, N., Lean, M. (2006). ABC of obesity Assessment of obesity and its clinical implications. BMJ (Clinical research ed.), 333, 695.

Instituto Nacional de Câncer. (2020). Quais os derivados do tabaco mais agressivos à saúde e como agem? https://www.inca.gov.br/perguntas-frequentes/quaisos-derivados-tabaco-mais-agressivos-saude-e-como-agem.

Instituto Nacional de Câncer. (2021). Tabagimo. Causas e Prevenção. https://www.inca.gov.br/tabagismo.

Ministério da Saúde. (2019). Secretária de Vigilância em Saúde. Vigitel Brasil 2018: vigilância de fatores de risco e proteção para doenças crônicas por inquérito telefônico.

Ministério da Saúde. (2011). Instituto Nacional de Câncer José de Alencar Gomes da Silva. A Situação do Tabagismo no Brasil. 76p.

Ministério da Saúde. (2018). Instituto Nacional de Câncer José de Alencar Gomes da Silva. Estudo associa fumar a aumento da circunferência abdominal entre adolescentes brasileiros. https://www.inca.gov.br/noticias/estudo-associa-fumar-aumento-da-circunferencia-abdominal-entre-adolescentes-brasileiros\#: :text= Tam b\%C3\%A9m\%20foi\%20lan\%C3\%A7ada\%20a\%20campanha,que\%20entre\%20os\%20n\%C3\%A3o\%20fumantes. Acesso em out. 2020.

Ministério da Saúde. (2020). Instituto Nacional de Câncer José de Alencar Gomes da Silva. Programa Nacional de Controle do Tabagismo. INCA, 2020. https://www.inca.gov.br/programa-nacional-de-controle-do-tabagismo/tabagismo.

Moreno, R., Moreno, D., \& Soares, M. (1999). Psicofarmacologia de antidepressivos. Revista Brasileira De Psiquiatria, 21(1), 24-40. https://doi.org/10.1590/s1516-44461999000500006

Kawada, T. M. D. (2016). Tabagismo, pressão arterial sistólica, glicose plasmática em jejum e progressão da aterosclerose carotídea. Nicotine \& Tobacco Research, 18, 1680

Kong, C. et al., Smoking is associated with increased hepatic lipase activity, insulin resistance, dyslipidaemia and early atherosclerosis in Type 2 diabetes. Atherosclerosis. 156, 373

Kumar, V., Abbas, A. K., Fausto, N. \& Aster, J. C (2010). Bases Patológicas das Doenças. (8a ed.), Elsevier. 1458p.

Laranjeira, R. \& Alves, A. G. H. Tratamento da Dependência da Nicotina. Centro de Estudos. Departamento de psiquiatria - UNIFESP/EPM. 34. http://www2.unifesp.br/dpsiq/polbr/ppm/atu1_02.htm 
Pereira, C. F. \& Vargas, D. (2015). Perfil de mulheres que realizaram tratamento para cessação do tabagismo: revisão sistemática. Revista de Saúde Pública, 39, $1-6$.

Pessoa, D. L. R., Santos, K. F. dos., Rodrigues, K. P., Dias, A. M. C., Souza, I. B. J. de., Gouveia, D. M. de., Ramos, A. S. M. B. . (2020). Reflexões sobre a associação entre o tabagismo e COVID - 19: revisão de literatura. Research, Society and Development, 9(9).

Pinto, M. F. T., Riviere, A. P., Biz, A., Schluckbier, L. \& Araújo, A. (2012) Carga das doenças tabaco-relacionadas para o Brasil. Relatório final. Rio de Janeiro: Fundação Oswaldo Cruz, 41 p.

Santos, C. B., Scortegagna, S. A., Franco, R. R. C. \& Wibelinger, L. M. (2019, agosto 28). Variáveis clínicas e razões para busca de tratamento de pacientes tabagistas. Revista Eletrônica Saúde Mental Alcool e Drogas (Edição em Português), 15, 77-86

Sarmento, K. K. F., Medeiros, K. M. de, Brito, Y. J. V. de, Medeiros, K. M. de, \& Lima, C. A. P. de. (2019, 20 de Novembro). O consumo de cigarros causando a dependência de nicotina. [Sessão de pôsteres] I Congresso Internacional de Meio Ambiente e Sociedade, Campina Grande.

Silvano, F. N. (2012). Tabagismo: Um Problema à Saúde Econômica da Sociedade. TCC (Graduação) - Curso de Economia e Relações Internacionais da Universidade Federal de Santa Catarina, Florianópolis. https://necat.paginas.ufsc.br/files/2011/10/Fernanda-Nadir-Silvano.pdf

Sousa, M. G. (2015). Tabagismo e Hipertensão arterial: como o tabaco eleva a pressão. Revista Brasileira de Hipertensão, $22,78-83$.

Tabagismo. INCA - Instituto Nacional de Câncer. (2021). https://www.inca.gov.br/tabagismo.

Tabagismo passivo. INCA - Instituto Nacional de Câncer. (2021). https://www.inca.gov.br/tabagismo/tabagismo-passivo.

Tratamento do tabagismo. INCA - Instituto Nacional do Câncer. (2021). https://www.inca.gov.br/en/node/1483.

Violante, L. M. D. (2011). O tabagismo e o peso corporal. 2011. 62 f. TCC (Graduação) - Curso de Medicina, Faculdade de Medicina da Universidade de Coimbra, Coimbra. https://estudogeral.uc.pt/bitstream/10316/80760/1/O\%20TABAGISMO\%20E\%20O\%20PESO\%20CORPORAL\%20final.pdf.

Vold, M. L., Aasebo, U. \& Melbye, H. Baixo FEV1, história de tabagismo e obesidade são fatores associados à diminuição da saturação de oxigênio em uma coorte da população adulta. Jornal Internacional de Doença Pulmonar Obstrutiva Crônica, 9, 1225-1233 